\title{
Use of subconjunctival injections of 5-fluorouracil to rescue and prolong intraocular pressure reduction for a failing Ahmed glaucoma implant
}

\author{
Kevin Kaplowitz ${ }^{1}$. James C. Tsai ${ }^{2}$ (D)
}

Received: 11 July 2017 / Accepted: 25 July 2017 /Published online: 11 August 2017

(C) Springer-Verlag GmbH Germany 2017

Dear Editor,

We thank Quaranta et al. for their insightful comments. We extend our profound apologies for failing to cite their study among the relevant papers that we reviewed in our discussion section [1]. Unfortunately, it appears that their publication was not in press at the time of our comprehensive literature search. Moreover, with respect to the ttest, we concur that our paper could have provided more statistical details and particularly specified that the paired t-test was used for within-group comparisons, and the unpaired t-test was used to compare the control patient group to the 5-fluorouracil group. We thank Quaranta et al. for bringing this to our attention, as well as highlighting the value of collecting more patient data in regards to postoperative subconjunctival injections with 5-fluorouracil (with or without associated needle revision procedure).
Compliance with ethical standards

Funding/support Supported in part by an unrestricted departmental grant from Research to Prevent Blindness, Inc., New York, NY (JCT).

Financial disclosures All authors report no proprietary or commercial interest for any product or concept discussed here.

Conflicts of interest No conflicts of interest.

\section{References}

1. Quaranta L, Floriani I, Hollander L, Poli D, Katsanos A, Konstas AG (2016) Needle revision with 5-fluorouracil for the treatment of Ahmed glaucoma valve filtering blebs: 5 -fluoruracil needling revision can be a useful and safe tool in the management of failing Ahmed glaucoma valve filtering blebs. J Glaucoma 25(4):e367e371

James C. Tsai

jtsai@nyee.edu

1 Loma Linda VA, Loma Linda University, 11201 Benton St, Loma, Linda, CA 92357, USA

2 Icahn School of Medicine at Mount Sinai, New York Eye and Ear Infirmary of Mount Sinai, 310 East 14th Street, New

York, NY 10003, USA 